EPJ Web of Conferences 75, 01004 (2014)

DOI: $10.1051 /$ epjconf/ 20147501004

(C) Owned by the authors, published by EDP Sciences, 2014

\title{
Co thin film with metastable bcc structure formed on GaAs(111) substrate
}

\author{
Shigeyuki Minakawa ${ }^{1, a}$, Daisuke Suzuki ${ }^{1}$, Mitsuru Ohtake $^{1}$, Nobuyuki Inaba ${ }^{2}$, and Masaaki Futamoto ${ }^{1}$ \\ ${ }^{1}$ Faculty of Science and Engineering, Chuo University, 1-13-27 Kasuga, Bunkyo-ku, Tokyo 112-8551, Japan \\ ${ }^{2}$ Faculty of Engineering, Yamagata University, 4-3-16 Jonan, Yonezawa, 992-8510 Yamagata, Japan
}

\begin{abstract}
Co thin films are prepared on $\operatorname{GaAs}(111)$ substrates at temperatures ranging from room temperature to $600{ }^{\circ} \mathrm{C}$ by radio-frequency magnetron sputtering. The growth behavior and the detailed resulting film structure are investigated by in-situ reflection high-energy electron diffraction and X-ray diffraction. In early stages of film growth at temperatures lower than $200{ }^{\circ} \mathrm{C}$, Co crystals with metastable $A 2$ (bcc) structure are formed, where the crystal structure is stabilized through hetero-epitaxial growth. With increasing the film thickness beyond $2 \mathrm{~nm}$, the metastable structure starts to transform into more stable $A 1$ (fcc) structure through atomic displacements parallel to the $A 2\{110\}$ close-packed planes. The crystallographic orientation relationship between the $A 2$ and the transformed $A 1$ crystals is $A 1\{111\}<1 \overline{1} 0>\| A 2\{110\}<001>$. When the substrate temperature is higher than $400{ }^{\circ} \mathrm{C}, \mathrm{Ga}$ atoms of substrate diffuse into the Co films and a Co-Ga alloy with bcc-based ordered structure of $B 2$ is formed.
\end{abstract}

\section{Introduction}

Cobalt $(\mathrm{Co})$ is a $3 d$ ferromagnetic transition metal which has two stable crystallographic phases, $A 3$ (hcp) and $A 1$ (fcc). $A 2$ (bcc) phase is metastable and does not appear in the bulk phase diagram. Recently, application of Co thin film with $A 2$ structure is providing a new possibility a new possibility in the development of magnetic devices. Tri-layer films consisting of $A 2-\mathrm{Co}$ and oxide $(\mathrm{MgO}$ or $\mathrm{SrTiO}_{3}$ ) layers are reported to show high tunnelling magnetoresistance ratios [1-3]. Understanding of the formation conditions is important in order to apply the $A 2-C o$ film for practical applications.

Formation of $A 2$ phase has been recognized for Co films of a few nm thicknesses grown on GaAs substrates of (100) [4-7] and (110) [4, 8, 9] orientations by molecular beam epitaxy. With increasing the thickness, most of the A2-Co crystals tended to transform into more stable $A 3$ or $A 1$ crystals. The film growth behavior and the film structure vary depending on the substrate orientation. However, there are very few reports on preparation of Co films on $\operatorname{GaAs}(111)$ substrates. In the present study, Co films are deposited on $\operatorname{GaAs}(111)$ substrates by magnetron sputtering, which is suitable for practical applications. The growth process and the detailed resulting film structure are investigated.

\section{Experimental procedure}

A radio-frequency (RF) magnetron sputtering system equipped with a reflection high-energy electron diffraction (RHEED) facility was used. The base pressures were lower than $4 \times 10^{-7} \mathrm{~Pa}$. Before film formation, $\operatorname{GaAs}(111)$ substrates were heated at $600{ }^{\circ} \mathrm{C}$ in the chamber to obtain clean surfaces. Figure 1(a-1) shows the RHEED pattern observed for a GaAs substrate after heating. A clear diffraction pattern from a clean B3(111) single-crystal surface shown in the schematic diagram of figure 1(a-2) is observed. A Co target of 3 in diameter was employed. The distance between target and substrate, the Ar gas pressure, and the RF power were respectively fixed at $150 \mathrm{~mm}, 0.67 \mathrm{~Pa}$, and $54 \mathrm{~W}$, where the deposition rate was $0.02 \mathrm{~nm} / \mathrm{s}$. Co films were deposited on the substrates at temperatures ranging between room temperature (RT) and $600{ }^{\circ} \mathrm{C}$. The thickness was varied in a range from 1 to $40 \mathrm{~nm}$.

The surface structure during sputter deposition process was studied by RHEED. The resulting film structure was investigated by $2 \theta / \omega$-scan out-of-plane, $2 \theta \chi / \varphi$-scan in-plane, and pole-figure X-ray diffractions (XRDs) with $\mathrm{Cu}-\mathrm{K} \alpha$ radiation $(\lambda=0.15418 \mathrm{~nm})$.

\section{Results and discussion}

Figures 1(b)-(f) show the RHEED patterns and the schematic diagrams of Co films of different thicknesses deposited at RT. A clear RHEED pattern corresponding to a diffraction pattern from $A 2(111)$ single-crystal surface with reconstructed structure of $p(3 \times 3)$ [figure $2(\mathrm{~d})]$ is observed for the 1 -nm-thick film [figure $1(\mathrm{~b}-1)]$. A Co single-crystal film with metastable $A 2$ structure is obtained in an early stage of film growth on GaAs(111) substrate, similar to the cases of films deposited on $\operatorname{GaAs}(100)$ [4-7] and $\operatorname{GaAs}(110)[4,8,9]$ substrates. The 

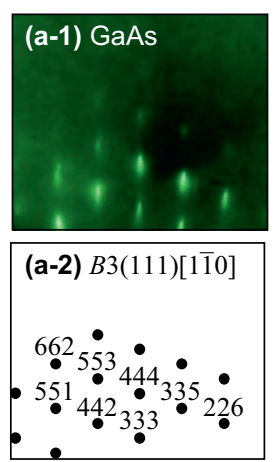
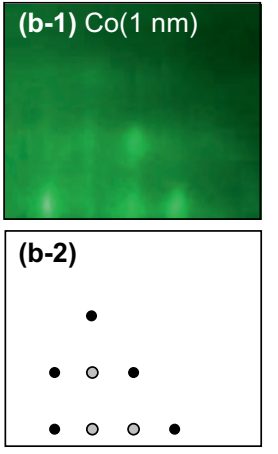
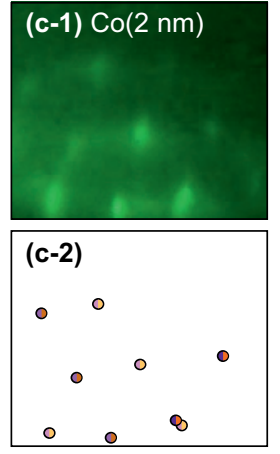
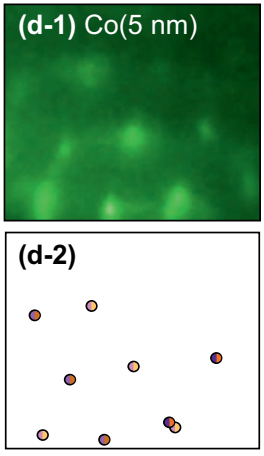
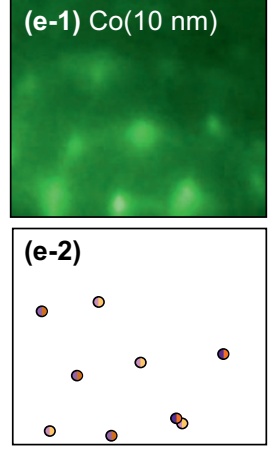
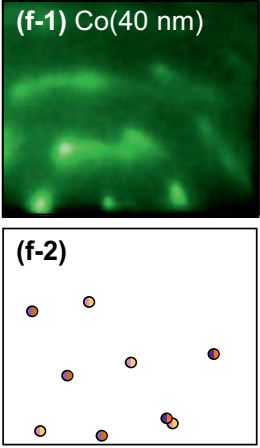

Fig. 1. (a-1) RHEED patterns observed for a GaAs(111) substrate after heating at $600{ }^{\circ} \mathrm{C}$. (a-2) Schematic diagram of RHEED pattern simulated for a B3(111) single-crystal surface. (b-1)-(f-1) RHEED patterns and (b-2)-(f-2) the schematic diagrams of Co films of (b) 1 , (c) 2, (d) 5, (e) 10, and (f) $40 \mathrm{~nm}$ thicknesses deposited on GaAs substrates at RT. The incident electron beam is parallel to GaAs[110].

(a) $A 2$

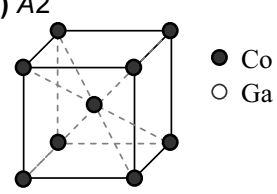

(b) $B 2$

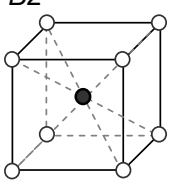

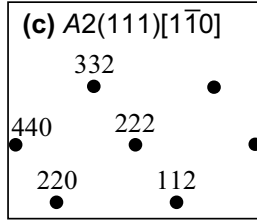
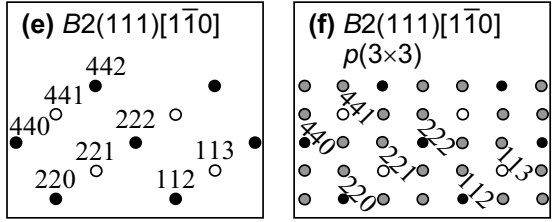

Fig. 2. (a, b) Crystal structures of (a) $A 2$ and (b) B2. (c)-(f) Schematic diagrams of RHEED patterns simulated for (c, d) $A 2(111)$ and $(\mathrm{e}, \mathrm{f}) B 2(111)$ single-crystal surfaces $(\mathrm{c}, \mathrm{e})$ without and $(\mathrm{d}, \mathrm{f})$ with reconstructed structure of $p(3 \times 3)$. The incident electron beam is parallel to [110]. The schematic diagrams are calculated by using a lattice constant of $a=0.28 \mathrm{~nm}$.

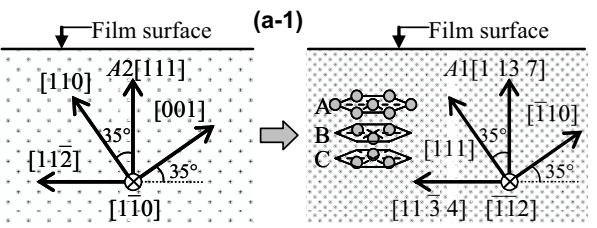

(b-1)
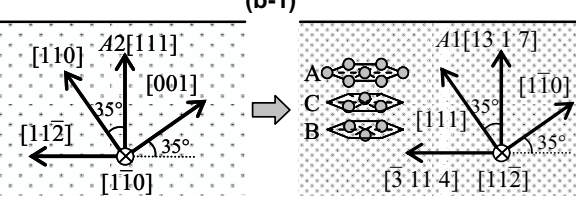

(c-1)
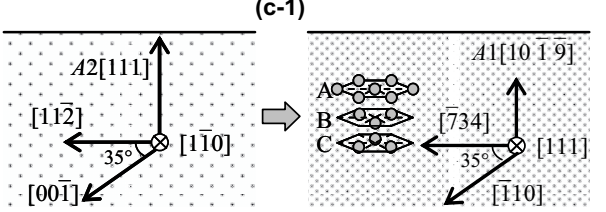

(d-1)

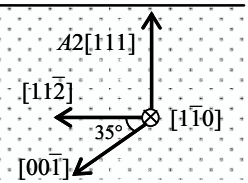

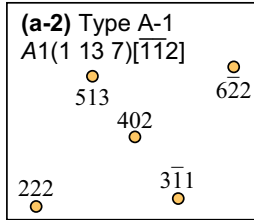
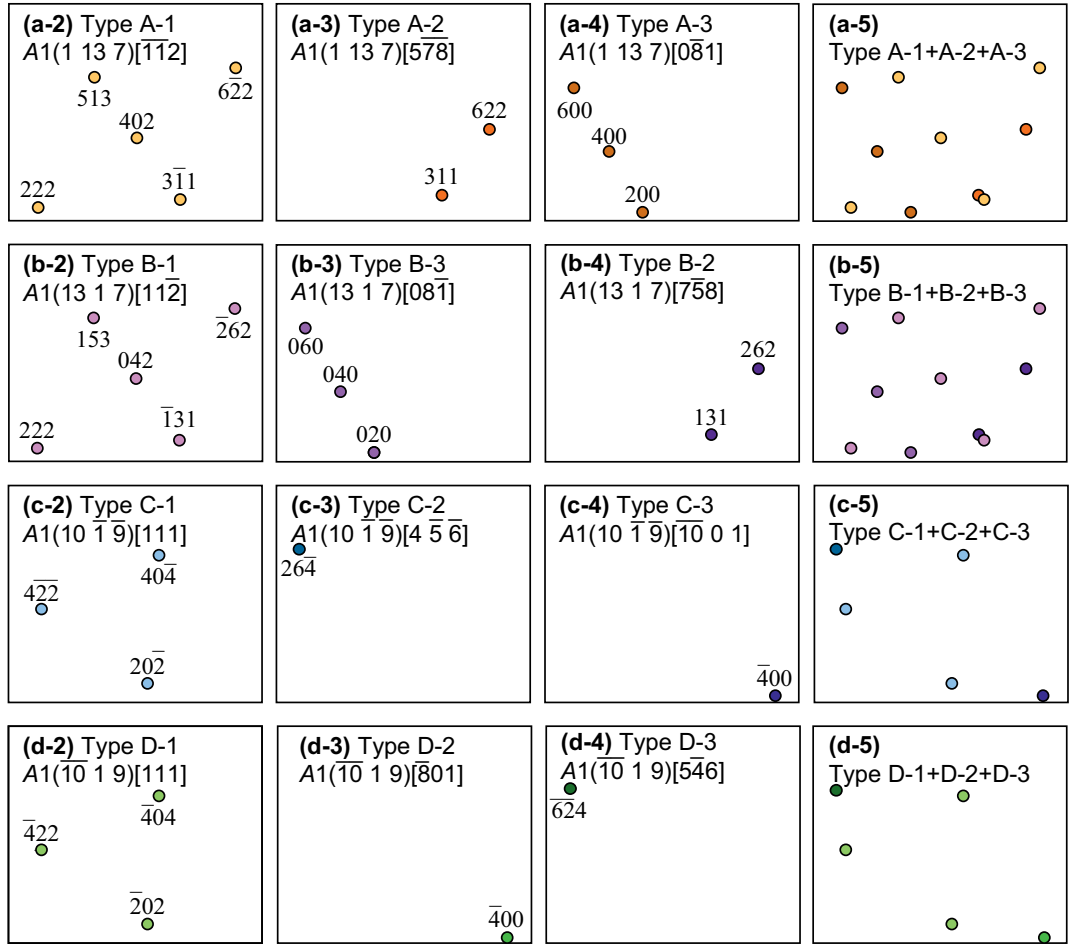
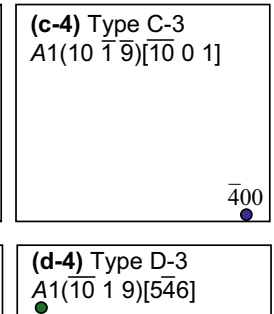

은

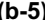

Type B-1+B-2+B-3
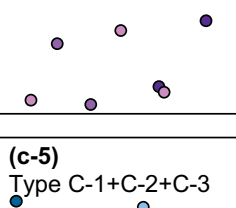

$\circ$

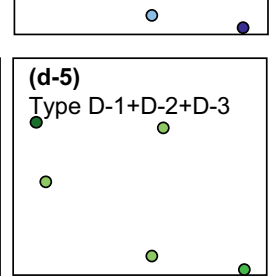

Fig. 3. (a-1)-(d-1) Schematic diagrams showing the $A 2-A 1$ phase transformation through atomic displacements parallel to (a-1, b-1) $A 2(110)$ and (c-1, d-1) $A 2(1 \overline{1} 0)$ planes. The stacking sequence of $A 1(111)$ is different between (a-1) and (b-1) and between (c-1) and (d-1). (a-2)-(d-4) Schematic diagrams of RHEED patterns simulated for (a-2)-(a-4) A1(1 13 7), (b-2)-(b-4) A1(13 1 7), (c-2)-(c-4) $A 1\left(\begin{array}{ll}10 & 1\end{array}\right)$, and (d-2)-(d-4) $A 1\left(\begin{array}{lll}10 & 1 & 9\end{array}\right)$ crystals transformed from $A 2$ structure through atomic displacements parallel to (a-2, b-2) $A 2(110),(\mathrm{a}-3, \mathrm{~b}-3) A 2(101),(\mathrm{a}-4, \mathrm{~b}-4) A 2(011),(\mathrm{c}-2, \mathrm{~d}-2) A 2(1 \overline{1} 0),(\mathrm{c}-3, \mathrm{~d}-3) A 2(10 \overline{1})$, and (c-4, d-4) $A 2(0 \overline{1} 1)$ planes. The incident

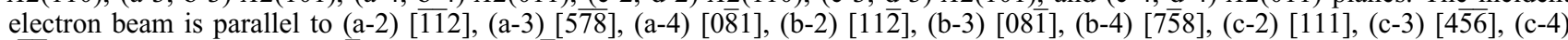
[10 0 1], (d-2) [111], (d-3) [801], or (d-4) [546]. The schematic diagrams are calculated by using a lattice constant of $a=0.35 \mathrm{~nm}$. Schematic diagrams of (a-5)-(d-5) are drawn by overlapping (a-2)-(d-2), (a-3)-(d-3), and (a-4)-(d-4), respectively. 
(a) $A 1\left(\begin{array}{lll}1 & 13 & 7\end{array}\right)$

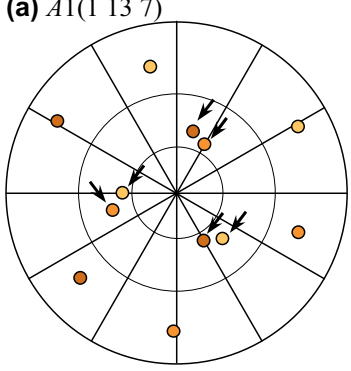

(b) $A 1\left(\begin{array}{lll}13 & 1 & 7\end{array}\right)$

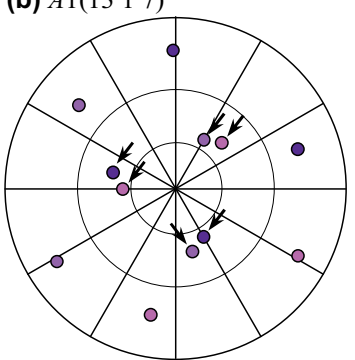

(c) $A 1(\overline{1} 10 \overline{9})$

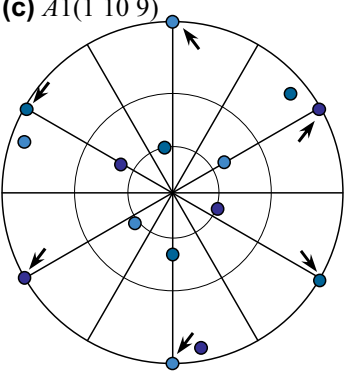

(d) $A 1(1 \overline{10} 9)$

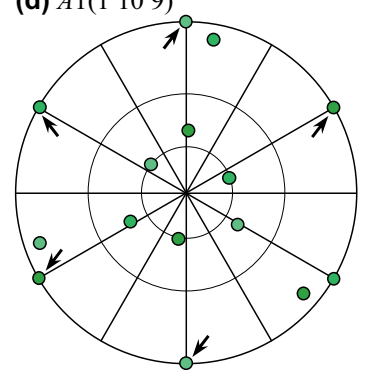

(e)

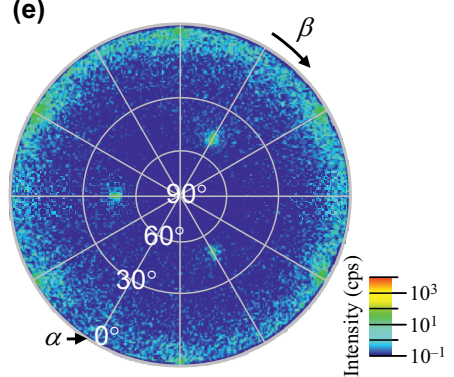

(f)

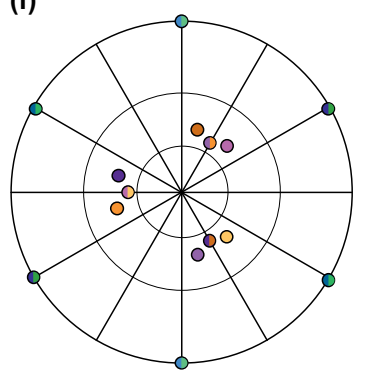

Fig. 4. (a)-(d) Schematic diagrams of pole-figure XRD patterns simulated for (a) $A 1\left(\begin{array}{llll}1 & 13 & 7\end{array}\right)$, (b) $A 1\left(\begin{array}{ll}13 & 1\end{array}\right)$, (c) $A 1(\overline{1} 10 \overline{9})$, and (d)

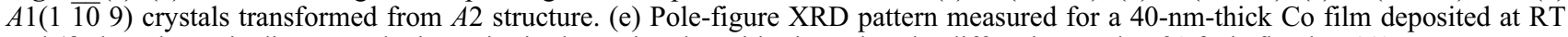
and (f) the schematic diagram. The intensity is shown in a logarithmic scale. The diffraction angle of $2 \theta B$ is fixed at $44^{\circ}$.

epitaxial orientation relationship is determined as

$$
\operatorname{Co}(111)[1 \overline{1} 0]_{A 2} \| \operatorname{GaAs}(111)[1 \overline{1} 0]_{B 3} \text {. }
$$

With increasing the thickness up to $2 \mathrm{~nm}$ [figure 1(c)], RHEED spots other than the spots from $A 2$ crystal appear. The pattern is analyzed to be an overlap of reflections from three types of $A 1\left(\begin{array}{lll}1 & 13 & 7\end{array}\right)$ and $A 1\left(\begin{array}{lll}13 & 1 & 7\end{array}\right)$ crystals. The $A 2$ structure is transforming into more stable $A 1$ structure. The crystallographic orientation relationships between $A 2$ and $A 1$ crystals are determined by RHEED as follows,

$$
\begin{aligned}
& A 1(111)[\overline{1} 10] \| A 2(110)[001] \text { (type } \mathrm{A}-1), \\
& A 1(111)[\overline{1} 0] \| A 2(101)[0 \overline{1} 0] \text { (type } \mathrm{A}-2), \\
& A 1(111)[\overline{1} \overline{1} 2] \| A 2(011)[0 \overline{1} 1] \text { (type } \mathrm{A}-3), \\
& A 1(111)[\overline{1} 0] \| A 2(110)[001] \text { (type } \mathrm{B}-1), \\
& A 1(111)[\overline{1} 10] \| A 2(101)[0 \overline{1} 0] \text { (type } \mathrm{B}-2), \\
& A 1(111)[11 \overline{1}] \| A 2(011)[0 \overline{1} 1] \text { (type B-3). }
\end{aligned}
$$

These relationships are similar to the NishiyamaWasserman orientation relationship $[10,11]$. In these configurations, the close-packed planes of $A 2(110)$, $A 2(101)$, and $A 2(011)$, which are $55^{\circ}$ inclined from the in-plane, are parallel to the A2(111) closepacked plane, as shown for example in figures 3(a-1) and (b-1). The phase transformation is taking place through atomic displacements parallel to the closepacked planes. The stacking sequence of $A 1(111)$ is different between the types of A-1 and B-1, of A-2 and B-2, and of A-3 and B-3. Therefore, the $A 1$ crystals with types A-1, A-2, and A-3 possess a (1 13 7) plane parallel to the substrate surface, whereas those with types B-1, B-2, and B-3 possess a (13 1 7) plane parallel to the substrate surface. Furthermore, there is a possibility that the transformation is also taking place through atomic displacements parallel to the $A 2(1 \overline{1} 0)$, $A 2(10 \overline{1})$, and $A 2(0 \overline{1} 1)$ close-packed planes which are perpendicular to the substrate surface, as shown in figures 3(c-1) and (d-1). The possible crystallographic orientation relationships are

$$
A 1(111)[1 \overline{1} 0] \| A 2(1 \overline{1} 0)[00 \overline{1}] \text { (type C-1), }
$$

$$
\begin{aligned}
& A 1(111)[\overline{1} 10] \| A 2(10 \overline{1})[010] \text { (type C-2), } \\
& A 1(111)[11 \overline{2}] \| A 2(0 \overline{1} 1)[0 \overline{1} \overline{1}] \text { (type C-3), } \\
& A 1(111)[\overline{1} 10] \| A 2(1 \overline{1} 0)[00 \overline{1}] \text { (type D-1), } \\
& A 1(111)[1 \overline{1} 0] \| A 2(10 \overline{1})[010] \text { (type D-2), } \\
& A 1(111)[\overline{1} \overline{1} 2] \| A 2(0 \overline{1} 1)[0 \overline{1} \overline{1}] \text { (type D-3). }
\end{aligned}
$$

Figures 3(c-5) and (c-6) show the schematic diagrams of diffraction patterns simulated for $A 1$ crystals transformed in the orientation relationships of types $\mathrm{C}$ and $\mathrm{D}$, respectively. However, these diffraction patterns are not clearly recognized in the RHEED pattern observed for the 2-nm-thick Co film [figure 1(c)]. However, the transformations of types $\mathrm{C}$ and D may occur mainly within the film, since the RHEED detects a crystallographic information around the film surface. In order to confirm the existence of $A 1$ crystals with types $\mathrm{C}$ and $\mathrm{D}$, polefigure XRD analysis, which is described later, was carried out. As the thickness increases beyond $5 \mathrm{~nm}$ [figures 1(d)-(f)], the RHEED spots become broader. The result indicates a possibility that the crystallographic defects like planar faults (stacking faults) are involved. Figures 4(a)-(d) show the schematic diagrams of polefigure XRD patterns simulated for the transformed $A 1$ crystals with the types A, B, C, and D. Here, the diffraction angle of $2 \theta B$ is fixed at $44^{\circ}$, where $A 1\{111\}$ reflections are expected to be detectable. Figures $4(\mathrm{e})$ and (f) show the pole-figure XRD pattern measured for the 40-nm-thick Co film deposited at RT and the schematic diagram, respectively. Reflections of three-fold symmetry, which originate from the $A 1$ crystals with types $\mathrm{C}$ and $\mathrm{D}$, are observed around the tilt angle, $\alpha$, of $55^{\circ}$. Furthermore, reflections of six-fold symmetry, which originate from $A 1$ crystals with types $\mathrm{C}$ and $\mathrm{D}$, are recognized around $\alpha=$ $0^{\circ}$. The result apparently shows that the transformation occurs not only along the directions of $A 1[110], A 1[101]$, and $A 1[011]$ which is $55^{\circ}$ canted from the substrate surface but also along the in-plane directions of [1 $1 \overline{1} 0]$,

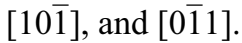

Figure 5(a) shows the RHEED patterns observed for 

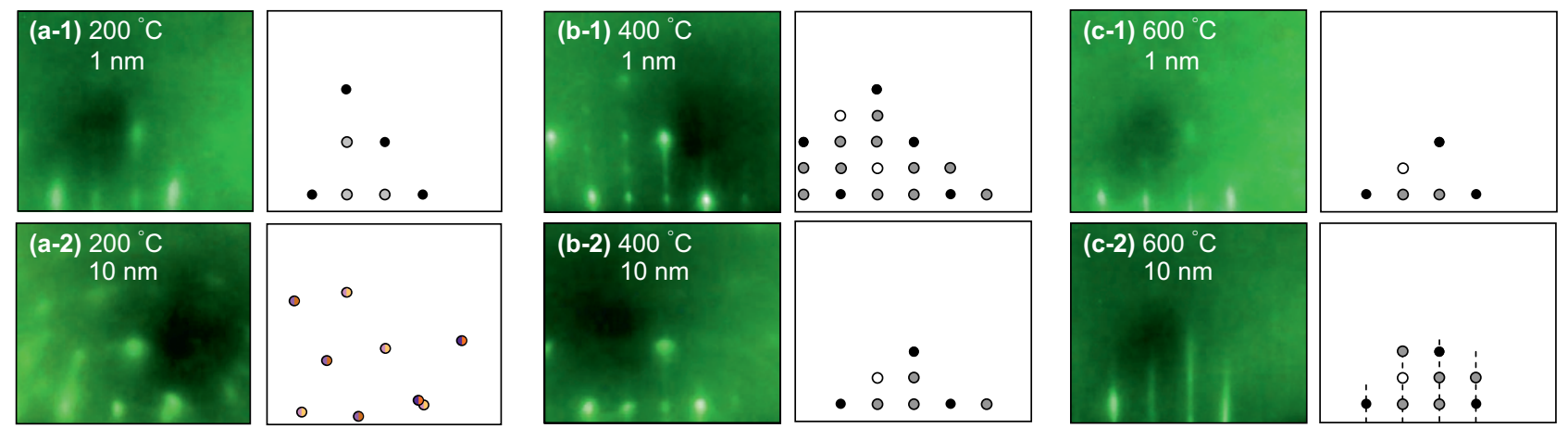

Fig. 5. RHEED patterns and the schematic diagrams of Co films of (a-1)-(c-1) 1 and (a-2)-(c-2) 10 nm thicknesses deposited at (a) 200 , (b) 400 , and (c) $600^{\circ} \mathrm{C}$. The incident electron beam is parallel to $\mathrm{GaAs}[1 \overline{1} 0]$.

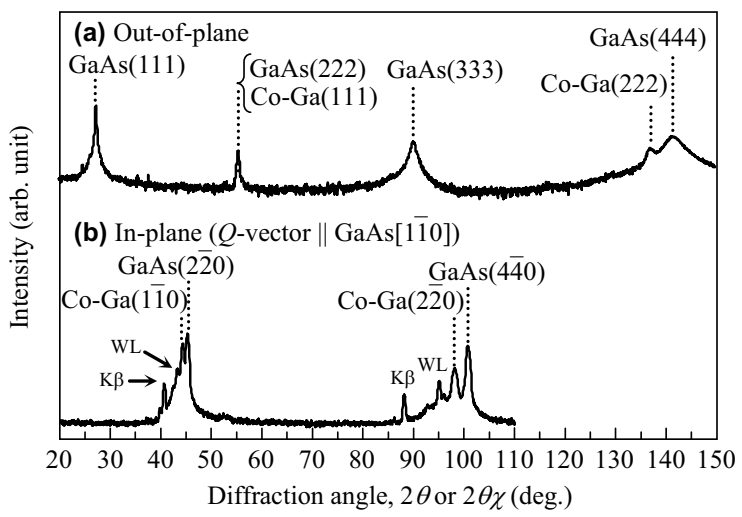

Fig. 6. (a) Out-of-plane and (b) in-plane XRD patterns of a Co film of $10 \mathrm{~nm}$ thickness deposited on $\operatorname{GaAs}(111)$ substrate at $600{ }^{\circ} \mathrm{C}$. The scattering vector of in-plane XRD is parallel to GaAs[110]. The intensity is shown in a linear scale. The small reflections noted as $\mathrm{K} \beta$ and $\mathrm{WL}$ are due to $\mathrm{Cu}-\mathrm{K} \beta$ and $\mathrm{W}-\mathrm{L} \alpha$ radiations included in the $\mathrm{X}$-ray source, respectively.

Co films deposited at $200{ }^{\circ} \mathrm{C}$. A diffraction pattern corresponding to $A 2(111)$ texture is observed for the 1nm-thick film [figure 5(a-1)]. With increasing the thickness [figure 5(a-2)], a diffraction pattern from $A 1$ crystals transformed from $A 2$ structure is observed. The nucleation and the transformation behaviours are similar to the case of film formation at RT.

Figures 5(b) and (c) show the RHEED patterns observed for the Co films deposited at 400 and $600{ }^{\circ} \mathrm{C}$, respectively. Clear diffraction patterns involving superlattice spots shown by the white circles in the schematic diagrams are observed. The RHEED patterns correspond to a formation of (111) crystal with bcc-based ordered structure of $B 2$. It is considered that $\mathrm{Ga}$ atoms of substrate diffuse into the film and a Co-Ga alloy crystal with $B 2$ structure is formed on the substrate. The crystallographic orientation relationship is determined as

$$
\text { Co-Ga(111)[1] } 10]_{B 2} \| \operatorname{GaAs}(111)[1 \overline{1} 0]_{B 3} \text {. }
$$

Figure 6 shows the out-of-plane and in-plane XRD patterns measured for the 10-nm-thick Co film deposited at $600{ }^{\circ} \mathrm{C}$. The in-plane pattern is measured by making the scattering vector parallel to $\mathrm{GaAs}[1 \overline{1} 0]$. $\mathrm{Co}-\mathrm{Ga}(111)$ and $\mathrm{Co}-\mathrm{Ga}(222)$ out-of-plane and $\mathrm{Co}-\mathrm{Ga}(1 \overline{10})$ and Co$\mathrm{Ga}(2 \overline{2} 0)$ in-plane reflections are observed in addition to reflections from GaAs substrate. It is necessary to employ a low substrate temperature to prepare an $A 2-\mathrm{Co}$ film on a GaAs substrate.

\section{Conclusion}

Co thin films are prepared on GaAs(111) substrates by varying the thickness from 1 to $40 \mathrm{~nm}$ and the substrate temperature from RT to $600{ }^{\circ} \mathrm{C}$. The growth behavior and the film structure are studied by RHEED and XRD. Co crystals with $A 2$ structure nucleate epitaxially on the substrates at temperatures lower than $200{ }^{\circ} \mathrm{C}$. With increasing the thickness, the $A 2$ structure transforms into more stable $A 1$ structure through atomic displacements parallel to the $A 2\{110\}$ close-packed planes. When the substrate temperature increases beyond $400{ }^{\circ} \mathrm{C}$, Ga atoms diffuse from the substrate into the Co film and a Co-Ga alloy film with $B 2$ ordered structure is formed.

\section{Acknowledgements}

A part of this work was supported by JSPS KAKENHI Grant Number 25420294, JST A-STEP Grant Number AS242Z00169M, and Chuo University Grant for Special Research.

\section{References}

1. X. -G. Zhang, W. H. Butler, Phys. Rev. B 70, 172407 (2004)

2. J. P. Velev, K. D. Belashchenko, D. A. Stewart, M. van Schifgaarde, S. S. Jaswal, E. Y. Tsymbal, Phys. Rev. Lett. 95, 216601 (2005)

3. S. Yuasa, A. Fukushima, H. Kubota, Y. Suzuki, K. Ando, Appl. Phys. Lett. 89, 042505 (2006)

4. F. Xu, J. J. Joyce, M. W. Ruckman, H. -W. Chen, F. Boscherini, D. M. Hill, S. A. Chambers, J. H. Weaver, Phys. Rev. B 35, 2375 (1987)

5. Y. U. Idzerda, B. T. Jonker, W. T. Elam, G. A. Prinz, J. Vac. Sci. Technol. A 8, 1572 (1990)

6. S. J. Blundell, M. Gester, J. A. C. Bland, C. Daboo, E. Gu, M. J. Baird, A. B. Rives, J. Appl. Phys. 73, 5948 (1993)

7. Y. Z. Wu, H. F. Ding, C. Jing, D. Wu, G. L. Liu, V. Gordon, G. S. Dong, X. F. Jing, Phys. Rev. B 57, 11935 (1998)

8. G. A. Prinz, Phys. Rev. Lett, 54, 1051 (1985)

9. C. M. Teodorescu, M. G. Martin, N. Franco, H. Ascolani, J. Chrost, J. Avila, and M. C. Asensio, J. Electron Spectrosc. Relat. Phenom. 101-103, 493 (1999)

10. G. Wasserman, Arch. Eisenhuettenwes 16, 647 (1933)

11. Z. Nishiyama, Sci. Rep. Tohoku Univ. 23, 638 (1934) 